\title{
Lie Algebroids in the Loday-Pirashvili Category ${ }^{\star}$
}

Ana ROVI $\ddagger$

$\dagger$ School of Mathematics and Statistics, University of Glasgow, University Gardens, Glasgow G12 8QW, UK

$\ddagger$ School of Mathematics and Statistics, Newcastle University, Newcastle upon Tyne NE1 7RU, UK

E-mail: ana.rovi-garcia@ncl.ac.uk

URL: https://www.staff.ncl.ac.uk/ana.rovi-garcia/

Received February 27, 2015, in final form September 28, 2015; Published online October 02, 2015 http://dx.doi.org/10.3842/SIGMA.2015.079

\begin{abstract}
We describe Lie-Rinehart algebras in the tensor category $\mathcal{L M}$ of linear maps in the sense of Loday and Pirashvili and construct a functor from Lie-Rinehart algebras in $\mathcal{L M}$ to Leibniz algebroids.
\end{abstract}

Key words: Lie algebroid; Leibniz algebra; Courant algebroid; Leibniz algebroid 2010 Mathematics Subject Classification: 17A32; 53D17

\section{Introduction}

Leibniz algebras were introduced by Loday and Pirashvili [17, 18] as a non skew-symmetric generalisation of Lie algebras. Leibniz algebras appear in differential geometry as the algebraic structure on Courant algebroids [16] and in mathematical physics, for example in Chern-Simons theory [5].

Loday and Pirashvili [18] observed that Leibniz algebras can be described as the canonical map $\pi: \mathfrak{g} \rightarrow \mathfrak{g}_{\text {Lie }}$ where $\mathfrak{g}$ is a Leibniz algebra and $\mathfrak{g}_{\text {Lie }}$ is the Lie algebra which arises as the quotient of $\mathfrak{g}$ by the Leibniz ideal generated by elements $[x, x]_{\mathfrak{g}}$ for $x \in \mathfrak{g}$. This observation leads to their definition [19] of the monoidal category $\mathcal{L} \mathcal{M}$ of linear maps and to the construction of a pair of adjoint functors between Lie algebras and Leibniz algebras. Note that the category $\mathcal{L} \mathcal{M}$ can be seen as the category of truncated chain complexes of length one.

In this paper, we focus on the interplay between Lie-Rinehart algebras [8] (Lie algebroids [20] in a differential geometric context) and Leibniz algebroids [1, 10] which are generalisations of Lie algebras and Leibniz algebras respectively. Our goal is not to define a differential geometric counterpart of Loday and Pirashvili's category of linear maps but to use their construction to describe (and understand) some interesting relations between Lie-Rinehart algebras and Leibniz algebras. In this sense, we describe Lie-Rinehart algebras in $\mathcal{L} \mathcal{M}$ and then construct a functor from Lie-Rinehart algebras in $\mathcal{L} \mathcal{M}$ to Leibniz algebroids.

Throughout, let $R$ be a unital commutative ring and let an unadorned $\otimes$ denote $\otimes_{R}$.

Theorem 1.1. Let $(M \stackrel{g}{\rightarrow} A)$ be a commutative R-algebra object and $(N \stackrel{f}{\rightarrow} L)$ be a Lie algebra object in $\mathcal{L} \mathcal{M}$. A pair $((M \stackrel{g}{\rightarrow} A),(N \stackrel{f}{\rightarrow} L))$ is a Lie-Rinehart algebra object in $\mathcal{L} \mathcal{M}$ if

- $(A, L)$ is a Lie-Rinehart algebra with anchor $\rho_{0}: L \rightarrow \operatorname{Der}_{R}(A)$,

- the A-bimodule $M$ is a left $(A, L)$-module with action given by $\rho_{2}: L \rightarrow \operatorname{Hom}_{R}(M, M)$,

${ }^{\star}$ This paper is a contribution to the Special Issue on Poisson Geometry in Mathematics and Physics. The full collection is available at http://www.emis.de/journals/SIGMA/Poisson2014.html 
- the right $L$-module $N$ with action $N \otimes L \rightarrow N$ given by $n \otimes \xi \mapsto[n, \xi]$ for all $n \in N, \xi \in L$ is also a left $A$-module with $[-,-]$ satisfying

$$
[a \cdot n, \xi]=a \cdot[n, \xi]-\rho_{0}(\xi)(a) \cdot n, \quad \forall a \in A,
$$

- both $f$ and $g$ are L-equivariant and A-linear,

and there exist

- an A-module map $\lambda: M \otimes_{A} L \rightarrow N$,

- an A-module map $\rho_{1}: N \rightarrow \operatorname{Der}_{R}(A, M)$ satisfying

$$
g\left(\rho_{1}(n)(a)\right)=\rho_{0}(f(n))(a), \quad \rho_{1}([n, \xi])=\left[\rho_{1}(n),\left(\rho_{0}+\rho_{2}\right)(\xi)\right]
$$

for all $a \in A, m \in M, n \in N, \xi \in L$.

Our second result consists in the construction of a functor from the category of Lie-Rinehart algebra objects in $\mathcal{L} \mathcal{M}$ to the category of Leibniz algebroids.

Theorem 1.2. For a Lie-Rinehart algebra $((M \stackrel{g}{\rightarrow} A),(N \stackrel{f}{\rightarrow} L))$ in $\mathcal{L} \mathcal{M}$, the pair $(A, M \oplus N)$ is a Leibniz algebroid with anchor

$$
\rho_{M \oplus N}:=-\rho_{0} \circ f: M \oplus N \longrightarrow \operatorname{Der}_{R}(A)
$$

and Leibniz bracket on the $A$-module $M \oplus N$ given by

$$
\left[m_{1}+n_{1}, m_{2}+n_{2}\right]_{M \oplus N}:=-\rho_{2}\left(f\left(n_{2}\right)\right)\left(m_{1}\right)+\left[n_{1}, f\left(n_{2}\right)\right] .
$$

for all $m_{1}, m_{2} \in M$ and $n_{1}, n_{2} \in N$.

Since a very rich class of examples of Hopf algebroids [3, 13, 22] is the enveloping algebra of Lie-Rinehart algebras, following the argument given by Loday and Pirashvili in [19], we would expect a similar relation between the enveloping algebra of a Leibniz algebroid and Hopf algebroids in $\mathcal{L} \mathcal{M}$. However this generalisation of [19] requires not only the definition of a correct notion of enveloping algebra of a Leibniz algebroid (not yet defined in the literature), but also the construction of a functor from the category of Leibniz algebroids to the category of Lie-Rinehart algebras objects in $\mathcal{L M}$, which goes beyond the goals of this paper.

\section{Leibniz algebroids}

In this section we first recall the definitions of Leibniz algebras as given by Loday and Pirashvili [17, 18]. Secondly, we discuss Leibniz algebroids, see [10] for a differential geometric description, and give some motivating examples.

\subsection{Leibniz algebras}

Leibniz algebras were first defined by Blokh [2], later rediscovered and more intensively studied since $[4,18]$. For motivation, definitions and basic examples see $[17,18]$.

Definition 2.1. A right Leibniz algebra $\mathfrak{g}$ is an $R$-module equipped with a bilinear map, called the right Leibniz bracket and denoted by $[-,-]_{\mathfrak{g}}: \mathfrak{g} \otimes \mathfrak{g} \longrightarrow \mathfrak{g}$ which satisfies the identity

$$
\left[x,[y, z]_{\mathfrak{g}}\right]_{\mathfrak{g}}-\left[[x, y]_{\mathfrak{g}}, z\right]_{\mathfrak{g}}+\left[[x, z]_{\mathfrak{g}}, y\right]_{\mathfrak{g}}=0, \quad \text { for all } x, y, z \in \mathfrak{g} .
$$


Correspondingly, a left Leibniz algebra structure $[-,-]$ on an $R$-module $V$ is defined by $[x, y]:=[y, x]_{\mathfrak{g}}$ for $x, y \in V$ where $[-,-]_{\mathfrak{g}}$ satisfies $(2.1)$, see $[17,18]$.

Since Loday and Pirashvili use right Leibniz algebra structures in their work [17, 18, 19], we choose right Leibniz algebras as well, which we call from now on Leibniz algebras.

Remark 2.2. For a Leibniz algebra $\mathfrak{g}$ there exists a corresponding Lie algebra, denoted by $\mathfrak{g}_{\text {Lie }}$ and called the reduced Lie algebra of $\mathfrak{g}$, which arises by taking the quotient of $\mathfrak{g}$ by the Leibniz ideal generated by elements $[x, x]_{\mathfrak{g}} \in \mathfrak{g}$ for $x \in \mathfrak{g}$. Hence there exists a surjective map

$$
\pi: \mathfrak{g} \longrightarrow \mathfrak{g}_{\text {Lie }} \text {. }
$$

Example 2.3 (see [15]). Let $L$ be a Lie algebra over $R$ with bracket $[-,-]_{L}$. The bracket on the second tensor power of $L$ given by

$$
\left[x_{1} \otimes y_{1}, x_{2} \otimes y_{2}\right]_{L \otimes L}=\left[x_{1},\left[x_{2}, y_{2}\right]_{L}\right]_{L} \otimes y_{1}+x_{1} \otimes\left[y_{1},\left[x_{2}, y_{2}\right]_{L}\right]_{L}
$$

for all $x_{1}, x_{2}, y_{1}, y_{2} \in L$ endows $L \otimes L$ with a Leibniz algebra structure.

In the following example we reformulate the construction of the hemi-semi-direct product for left Leibniz algebras introduced by Kinyon and Weinstein in [11, Example 2.2] and endow the direct sum of a Lie algebra $L$ and a (left) $L$-module $V$ with a (right) Leibniz algebra structure.

Example 2.4. Let $L$ be a Lie algebra over $R$ and $V$ be a $L$-module with left action $L \otimes V \rightarrow V$ given by $\xi \otimes a \mapsto \xi(a)$ for all $a \in V$ and $\xi \in L$. The direct sum (of $R$-modules) $V \oplus L$ together with the bracket

$$
[a+\xi, b+\zeta]_{V \oplus L}:=\zeta(a)-[\xi, \zeta]_{L}, \quad a, b \in V, \xi, \zeta \in L
$$

becomes a (right) Leibniz algebra since the identity (2.1) is satisfied

$$
\begin{aligned}
& {\left[a+\xi,[b+\zeta, c+\gamma]_{V \oplus L}\right]_{V \oplus L}-\left[[a+\xi, b+\zeta]_{V \oplus L}, c+\gamma\right]_{V \oplus L}+\left[[a+\xi, c+\gamma]_{V \oplus L}, b+\zeta\right]_{V \oplus L}} \\
& \quad=\left[a+\xi, \gamma(b)-[\zeta, \gamma]_{L}\right]_{V \oplus L}-\left[\zeta(a)-[\xi, \zeta]_{L}, c+\gamma\right]_{V \oplus L}+\left[\gamma(a)-[\xi, \gamma]_{L}, b+\zeta\right]_{V \oplus L} \\
& \quad=-[\zeta, \gamma]_{L}(a)+\left[\xi,[\zeta, \gamma]_{L}\right]_{L}-\gamma(\zeta(a))-\left[[\xi, \zeta]_{L}, \gamma\right]_{L}+\zeta(\gamma(a))+\left[[\xi, \gamma]_{L}, \zeta\right]_{L}=0 .
\end{aligned}
$$

Definition 2.5 (see [17]). Let $\mathfrak{g}$ and $\mathfrak{g}^{\prime}$ be Leibniz algebras. A map of Leibniz algebras $\varphi: \mathfrak{g} \rightarrow \mathfrak{g}^{\prime}$ is a homomorphism of $R$-modules satisfying $\varphi\left([x, y]_{\mathfrak{g}}\right)=[\varphi(x), \varphi(y)]_{\mathfrak{g}^{\prime}}$ for all $x, y \in \mathfrak{g}$.

Proposition 2.6. Let $\mathfrak{g}$ be a Leibniz algebra over $R$ and let $M$ be a left module over its reduced Lie algebra $\mathfrak{g}_{\text {Lie }}$ with left action $\mathfrak{g}_{\text {Lie }} \otimes M \rightarrow M$ given by $\pi(g) \otimes m \mapsto \pi(g)(m)$ for all $m \in M$ and $g \in \mathfrak{g}$. The direct sum (of $R$-modules) $M \oplus \mathfrak{g}$ together with the bracket

$$
\left[m_{1}+g_{1}, m_{2}+g_{2}\right]_{M \oplus \mathfrak{g}}:=-\pi\left(g_{2}\right)\left(m_{1}\right)+\left[g_{1}, g_{2}\right]_{\mathfrak{g}}, \quad m_{1}, m_{2} \in M, g_{1}, g_{2} \in \mathfrak{g}
$$

is a Leibniz algebra.

Proof. Since $\pi: \mathfrak{g} \rightarrow \mathfrak{g}_{\text {Lie }}$ is a map of Leibniz algebras, a straightforward computation identical to the one carried out in Example 2.4 yields that $[-,-]_{M \oplus \mathfrak{g}}$ satisfies (2.1) and is hence a Leibniz bracket.

\subsection{Leibniz algebroids and related structures}

While Lie algebras can be generalised to Lie-Rinehart algebras [6, 8, 21] (Lie algebroids [20] in differential geometric context), Leibniz algebras $[2,18]$ give rise to different algebraic objects: Leibniz algebroids, first defined in a differential geometric context in [10]; Loday algebroids [24]; Courant algebroids [16]; Courant-Dorfman algebras, a term coined by Roytenberg [23] to denote a structure encompassing both Courant algebroids [16] and Dorfman algebras. See [12] for a description of the historic development of these structures. 


\subsubsection{Leibniz algebroids}

We propose a definition of Leibniz algebroids in purely algebraic terms, following the definitions given by Rinehart [21] and later by Huebschmann [8] for Lie-Rinehart algebras as an algebraic description of Lie algebroids.

Definition 2.7. Let $A$ be a commutative $R$-algebra and $\mathcal{E}$ be a Leibniz algebra over $R$ with bracket $[-,-]_{\mathcal{E}}$. The pair $(A, \mathcal{E})$ is called a Leibniz algebroid if the Leibniz algebra $\mathcal{E}$ has a left $A$-module structure $\mu: A \otimes \mathcal{E} \rightarrow \mathcal{E}$ given by $a \otimes e \mapsto a \cdot e$ for all $a \in A$ and $e \in \mathcal{E}$, and there exists an $A$-linear Leibniz algebra antihomomorphism $\rho_{\mathcal{E}}: \mathcal{E} \rightarrow \operatorname{Der}_{R}(A)$, called the anchor, satisfying

$$
\left[a \cdot e_{1}, e_{2}\right]_{\mathcal{E}}=a \cdot\left[e_{1}, e_{2}\right]_{\mathcal{E}}+\rho_{\mathcal{E}}\left(e_{2}\right)(a) \cdot e_{1}, \quad \text { for } e_{1}, e_{2} \in \mathcal{E}, \quad a \in A .
$$

Example 2.8. A Lie-Rinehart algebra $(A, L)$, with anchor $\rho_{L}$, is a Leibniz algebroid with anchor $-\rho_{L}$.

Proposition 2.9. Let $(A, L)$ be a Lie-Rinehart algebra, with anchor $\rho_{L}$, and let $M$ be a left $(A, L)$-module with action $L \otimes M \rightarrow M$ given by $\xi \otimes m \mapsto \nabla_{\xi}^{\ell}(m)$. The pair $(A, M \oplus L)$ is a Leibniz algebroid with anchor

$$
\rho_{M \oplus L}(m+\xi):=-\rho_{L}(\xi)
$$

and bracket on the direct sum $M \oplus L$ given by the (negative) hemi-semi-direct product $[-,-]_{M \oplus L}$ of $M$ by $L$ with action $L \otimes M \rightarrow M$ (or equivalently $\left.L \rightarrow \operatorname{Hom}_{R}(M, M)\right)$ given by $\nabla^{\ell}$.

Proof. First note that $\left[m_{1}+\xi, m_{2}\right]_{M \oplus L}=0$ for all $m_{1}, m_{2} \in M, \xi \in L$. Now, since $M$ is an $(A, L)$-module with action $\nabla^{\ell}: L \otimes M \rightarrow M$ given by $m \otimes \xi \mapsto \nabla_{\xi}^{\ell}(m)$, we endow the direct sum $M \oplus L$ with the Leibniz bracket given in (2.3), that is

$$
\left[m_{1}+\xi, m_{2}+\zeta\right]_{M \oplus L}=-\nabla_{\zeta}^{\ell}\left(m_{1}\right)+[\xi, \zeta]_{L} .
$$

We now check that the map in (2.5) is an antihomomorphism

$$
\begin{aligned}
\rho_{M \oplus L}\left(\left[m_{1}+\xi, m_{2}+\zeta\right]_{M \oplus L}\right) & =\rho_{M \oplus L}\left(-\nabla_{\zeta}^{\ell}\left(m_{1}\right)+[\xi, \zeta]_{L}\right)=-\rho_{L}\left([\xi, \zeta]_{L}\right) \\
& =-\left[\rho_{L}(\xi), \rho_{L}(\zeta)\right]_{\operatorname{Der}_{R}(A)} \\
& =\left[\rho_{L}(\zeta), \rho_{L}(\xi)\right]_{\operatorname{Der}_{R}(A)}=\left[\rho_{M \oplus L}(\zeta), \rho_{M \oplus L}(\xi)\right]_{\operatorname{Der}_{R}(A)}
\end{aligned}
$$

Lastly, we check that the compatibility condition beween $[-,-]_{M \oplus L}$ and the $A$-module structure on $M \oplus L$ given in (2.4) is satisfied

$$
\begin{aligned}
{[a \cdot(m+\xi), \zeta]_{M \oplus L} } & =-\nabla_{\zeta}^{\ell}(a \cdot m)+[a \cdot \xi, \zeta]_{L} \\
& =-a \cdot \nabla_{\zeta}^{\ell}(m)-\rho_{L}(\zeta)(a) \cdot m+a \cdot[\xi, \zeta]_{L}-\rho_{L}(\zeta)(a) \cdot \xi \\
& =a \cdot\left(-\nabla_{\zeta}^{\ell}(m)+[\xi, \zeta]_{L}\right)-\rho_{L}(\zeta)(a) \cdot(m+\xi) \\
& =a \cdot[m+\xi, \zeta]_{M \oplus L}+\rho_{M \oplus L}(\zeta)(a) \cdot(m+\xi) .
\end{aligned}
$$

Note that the Leibniz rule for $[-,-]_{M \oplus L}$ given by

$$
\begin{aligned}
{[m+\xi, a \cdot \zeta]_{M \oplus L} } & =-\nabla_{a \cdot \zeta}^{\ell}(m)+[\xi, a \cdot \zeta]_{L}=-a \cdot \nabla_{\zeta}^{\ell}(m)+a \cdot[\xi, \zeta]_{L}+\rho_{L}(\xi)(a) \cdot \zeta \\
& =a \cdot[m+\xi, \zeta]_{M \oplus L}-\rho_{M \oplus L}(\xi)(a) \cdot \zeta
\end{aligned}
$$

for all $a \in A$ and $\xi, \zeta \in L$ implies that the Leibniz algebroid $(A, M \oplus L)$ is local in the sense of [1, Definition 3.4].

In general, the relations between Lie algebras and Leibniz algebras will not induce relations between corresponding Lie-Rinehart algebras and Leibniz algebroids. 
Example 2.10. Let $(A, L)$ be a Lie-Rinehart algebra, with anchor $\rho_{L}$. The pair $(A, L \otimes L)$ where $L \otimes L$ is the Leibniz algebra with bracket given by (2.2) will not be a Leibniz algebroid in general. Since $[-,-]_{L}$ satisfies the Leibniz rule (2.4), we have

$$
\begin{aligned}
& {\left[a \cdot x_{1} \otimes y_{1}, x_{2} \otimes y_{2}\right]_{L \otimes L}=\left[a \cdot x_{1},\left[x_{2}, y_{2}\right]_{L}\right]_{L} \otimes y_{1}+a \cdot x_{1} \otimes\left[y_{1},\left[x_{2}, y_{2}\right]_{L}\right]_{L}} \\
& \quad=a \cdot\left[x_{1},\left[x_{2}, y_{2}\right]_{L}\right]_{L} \otimes y_{1}-\rho_{L}\left(\left[x_{2}, y_{2}\right]_{L}\right)(a) \cdot x_{1} \otimes y_{1}+a \cdot x_{1} \otimes\left[y_{1},\left[x_{2}, y_{2}\right]_{L}\right]_{L} \\
& \quad=a \cdot\left(\left[x_{1},\left[x_{2}, y_{2}\right]_{L}\right]_{L} \otimes y_{1}+x_{1} \otimes\left[y_{1},\left[x_{2}, y_{2}\right]_{L}\right]_{L}\right)-\rho_{L}\left(\left[x_{2}, y_{2}\right]_{L}\right)(a) \cdot x_{1} \otimes y_{1} \\
& \quad=a \cdot\left[x_{1} \otimes y_{1}, x_{2} \otimes y_{2}\right]_{L \otimes L}-\rho_{L}\left(\left[x_{2}, y_{2}\right]\right)(a) \cdot x_{1} \otimes y_{1},
\end{aligned}
$$

but the map $\gamma\left(x_{2} \otimes y_{2}\right):=\rho_{L}\left(\left[x_{2}, y_{2}\right]_{L}\right)$ is not $A$-linear since $[-,-]_{L}$ is not, hence the pair $(A, L \otimes L)$ does not admit an anchor map induced by $\rho_{L}$.

From [19] we know that to each Leibniz algebra $\mathfrak{g}$ we can canonically associate a Lie algebra $\mathfrak{g}_{\text {Lie }}$ by taking the quotient of $\mathfrak{g}$ by the two-sided ideal $[x, x]$ for $x \in \mathfrak{g}$. This relation does not generalise to a canonical relation between Leibniz algebroids and Lie-Rinehart algebras, so that given a Leibniz algebroid $(A, \mathcal{E})$, the reduced Lie algebra $\mathcal{E}_{\text {Lie }}$ will not be compatible in general with $A$.

Proposition 2.11. Let $(A, \mathcal{E})$ be a Leibniz algebroid with anchor $\rho_{\mathcal{E}}$. If $\operatorname{Ker}(\pi)$ is an $A$-submodule of $\mathcal{E}$, then the pair $\left(A, \mathcal{E}_{\text {Lie }}\right)$ is a Lie-Rinehart algebra with anchor denoted by $\rho_{\mathcal{E}_{\text {Lie }}}$ and given by $-\rho_{\mathcal{E}}$.

Proof. First note that the anchor $\rho_{\mathcal{E}}$ descends to an $R$-linear map $\gamma_{\mathcal{E}_{\text {Lie }}}: \mathcal{E}_{\text {Lie }} \rightarrow \operatorname{Der}_{R}(A)$ since $\rho_{\mathcal{E}}\left([e, e]_{\mathcal{E}}\right)=\left[\rho_{\mathcal{E}}(e), \rho_{\mathcal{E}}(e)\right]_{\mathcal{E}}=0$ for all $e \in \mathcal{E}$. Let us assume that $\pi: \mathcal{E} \rightarrow \mathcal{E}_{\text {Lie }}$ is $A$-linear. Then we have

$$
\gamma_{\mathcal{E}_{\text {Lie }}}(a \cdot \pi(e))=\gamma_{\mathcal{E}_{\text {Lie }}}(\pi(a \cdot e))=\rho_{\mathcal{E}}(a \cdot e)=a \cdot \rho_{\mathcal{E}}(e)=a \cdot \gamma_{\mathcal{E}_{\text {Lie }}}(\pi(e)),
$$

so that $\gamma_{\mathcal{E}_{\text {Lie }}}: \mathcal{E}_{\text {Lie }} \rightarrow \operatorname{Der}_{R}(A)$ is $A$-linear. Since $\rho_{\mathcal{E}}$ is a antihomomorphism while the anchor of a Lie-Rinehart algebra is a homomorphism, we set $\rho_{\mathcal{E}_{\text {Lie }}}:=-\gamma_{\mathcal{E}_{\text {Lie }}}$ so that $\left(A, \mathcal{E}_{\text {Lie }}\right)$ is a LieRinehart algebra and the following diagram

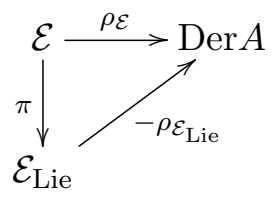

commutes.

Note that given a Leibniz algebroid $(A, \mathcal{E})$, the $A$-module structure on $\mathcal{E}$ will not descend to an $A$-module structure on the reduced Lie algebra $\mathcal{E}_{\text {Lie }}$ in general.

Example 2.12. On a manifold $M$, the bundle $E=T M \oplus T^{*} M$ has a natural Courant-Dorfman algebra structure with:

- a bilinear form given by $\langle X+\xi, Y+\zeta\rangle=\iota_{X} \zeta+\iota_{Y} \zeta$, where $X, Y \in T M, \xi, \zeta \in T^{*} M$ and $\iota_{X} \xi$ is the contraction of $X$ with $\xi$,

- a derivation $d: C^{\infty}(M) \rightarrow T^{*} X$ given by the differential of a function,

- a right Leibniz bracket given by $[X+\xi, Y+\zeta]=[X, Y]-\mathcal{L}_{X} \zeta+\mathcal{L}_{Y} \xi+d\left(\iota_{X} \zeta\right)$ where $[-,-]$ is the commutator of vector fields and $\mathcal{L}$ is the Lie derivative. 
Note that we have $[X+\xi, X+\xi]=d\left(i_{X} \xi\right)$ so that the reduced Lie algebra $E_{\text {Lie }}$ corresponding to $E=T M \oplus T^{*} M$ is

$$
E_{\text {Lie }}=T M \oplus T^{*} M /\langle\text { exact forms }\rangle
$$

with Lie bracket given by $[X+\xi, Y+\zeta]=[X, Y]-\mathcal{L}_{X} \zeta+\mathcal{L}_{Y} \xi$. In particular, we see that the map $\pi: E \rightarrow E_{\text {Lie }}$ is not $C^{\infty}(M)$-linear. Note also that while $[X+\xi, d f]=0$, we have

$$
[X+\xi, a \cdot d f]=-\langle X, d a\rangle \cdot d f+\langle X, d f\rangle \cdot d a .
$$

Note that the reduced Lie algebra $E_{\text {Lie }}$ is not an $C^{\infty}(M)$-module so that $\left(C^{\infty}(M), E_{\text {Lie }}\right)$ is not a Lie-Rinehart algebra.

\section{$3 \quad$ Lie-Rinehart algebras in the category $\mathcal{L} \mathcal{M}$ of linear maps}

Lie-Rinehart algebras [6, 8, 21] (Lie algebroids [20] in differential geometric context) were introduced by Herz [6] under the name Lie pseudo-algebra (also known as Lie algebroid [20] in a differential geometric context) and has been developed and studied as a generalisation of Lie algebras. The term Lie-Rinehart algebra was coined by Huebschmann [8], a term which acknowledges Rinehart's fundamental contributions [21] to the understanding of this structure. See [7, Section 1] for some historical remarks on this development.

We start this section by giving an overview of the category $\mathcal{L} \mathcal{M}$ as defined by Loday and Pirashvili [19]. In Section 3.2 we give the necessary tools and background to describe the universal algebra of derivations of an algebra in $\mathcal{L} \mathcal{M}$ (see Proposition 3.12). Lastly, in Section 3.3, we describe Lie-Rinehart algebras in the category $\mathcal{L} \mathcal{M}$ of linear maps.

\subsection{The category $\mathcal{L} \mathcal{M}$ of linear maps}

We first recall some fundamental concepts and definitions about the category $\mathcal{L} \mathcal{M}$ of linear maps, introduced by Loday and Pirashvili in [19], that are relevant for our main constructions later. We refer to [19] for further details. See [14] for results on Hopf algebras in $\mathcal{L} \mathcal{M}$.

Definition 3.1. The objects in the category $\mathcal{L} \mathcal{M}$ are $R$-module maps $(V \stackrel{u}{\rightarrow} W)$, where $u$ is called the vertical map. The morphisms between objects in $\mathcal{L} \mathcal{M}$ are pairs of maps $\mathrm{h}:=\left(h_{1}, h_{0}\right)$ such that the following diagram commutes:

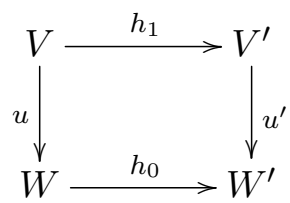

Given two morphisms $\mathrm{g}:=\left(g_{1}, g_{0}\right)$ and $\mathrm{h}:=\left(h_{1}, h_{0}\right)$ in $\mathcal{L} \mathcal{M}$, their composition $\mathrm{h} \circ \mathrm{g}$ is given by

$$
\mathrm{h} \circ \mathrm{g}=\left(h_{1}, h_{0}\right) \circ\left(g_{1}, g_{0}\right):=\left(h_{1} \circ g_{1}, h_{0} \circ g_{0}\right) \text {. }
$$

A morphism $\phi:=\left(\phi_{1}, \phi_{0}\right)$ is an isomorphism between objects $(V \stackrel{u}{\rightarrow} W)$ and $\left(V^{\prime} \stackrel{u^{\prime}}{\rightarrow} W^{\prime}\right)$ if and only if $\phi_{1}$ and $\phi_{2}$ are isomorphisms of $R$-modules.

Proposition 3.2. The category $\mathcal{L} \mathcal{M}$ is monoidal where the tensor product of two objects is

$$
(V \stackrel{u}{\rightarrow} W) \otimes\left(V^{\prime} \stackrel{u^{\prime}}{\rightarrow} W^{\prime}\right):=\left(V \otimes W^{\prime} \oplus W \otimes V^{\prime} \stackrel{u \otimes 1_{W^{\prime}}+1_{W} \otimes u^{\prime}}{\longrightarrow} W \otimes W^{\prime}\right),
$$

and the unit object is $(\{0\} \stackrel{0}{\rightarrow} R)$. Moreover, given two morphisms $\mathrm{g}:=\left(g_{1}, g_{0}\right)$ and $\mathrm{h}:=\left(h_{1}, h_{0}\right)$ in $\mathcal{L} \mathcal{M}$, their tensor product $\mathrm{g} \otimes \mathrm{h}$ is given by

$$
\mathrm{g} \otimes \mathbf{h}=\left(g_{1}, g_{0}\right) \otimes\left(h_{1}, h_{0}\right):=\left(g_{1} \otimes h_{0}+g_{0} \otimes h_{1}, g_{0} \otimes h_{0}\right) .
$$


Proof. See [19].

Furthermore, the monoidal category $\mathcal{L} \mathcal{M}$ is symmetric, with interchange morphism denoted by $\tau:(V \stackrel{u}{\rightarrow} W) \otimes\left(V^{\prime} \stackrel{u^{\prime}}{\rightarrow} W^{\prime}\right) \rightarrow\left(V^{\prime} \stackrel{u^{\prime}}{\rightarrow} W^{\prime}\right) \otimes(V \stackrel{u}{\rightarrow} W)$ and given by $\tau_{0}: W \otimes W^{\prime} \rightarrow W^{\prime} \otimes W$ and $\tau_{1}: V \otimes W^{\prime} \oplus W \otimes V^{\prime} \rightarrow V^{\prime} \otimes W \oplus W^{\prime} \otimes V$, see [19] for more details.

Commutative diagrams in $\mathcal{L} \mathcal{M}$ can be seen as commutative "cubes" in the category $R$-Mod.

Example 3.3. The commutative diagram in $\mathcal{L} \mathcal{M}$ given by

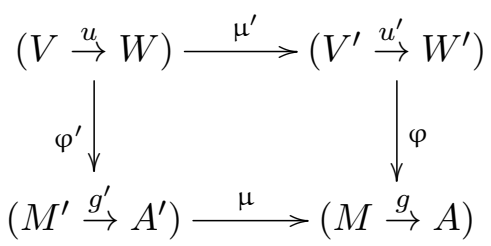

corresponds to the commuting "cube" given by

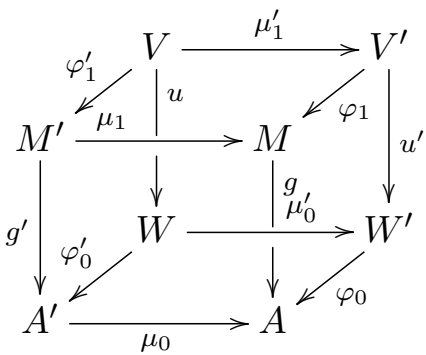

We now describe some of the fundamental algebraic structures in $\mathcal{L} \mathcal{M}$. For further details and proofs see [19].

\section{Proposition 3.4.}

- An associative algebra object $(M \stackrel{g}{\rightarrow} A)$ in $\mathcal{L} \mathcal{M}$ is a triple consisting of an associative $R$-algebra $A$, an A-bimodule $M$ and an A-bimodule map $g: M \rightarrow A$. Moreover, the algebra object $(M \stackrel{g}{\rightarrow} A)$ is commutative, if and only if the $A$-bimodule $M$ is symmetric and $A$ is commutative.

- A Lie algebra object $(N \stackrel{f}{\rightarrow} L)$ in $\mathcal{L} \mathcal{M}$ is equivalent to a Lie algebra $L$, a right $L$-module $N$ with right action $N \otimes L \rightarrow N$ given by $n \otimes \xi \mapsto[n, \xi]$ for all $n \in N$ and $\xi \in L$, and an R-linear L-equivariant map $f: N \rightarrow L$, i.e., $f([n, \xi])=[f(n), \xi]_{L}$.

Example 3.5. We give the following examples of objects in $\mathcal{L} \mathcal{M}$ :

- The surjective map $\pi: \mathcal{E} \rightarrow \mathcal{E}_{\text {Lie }}$ is a Lie algebra object in $\mathcal{L} \mathcal{M}$.

- Let $I$ be a two-sided ideal in an associative algebra $A$. The identity map id: $I \rightarrow A$ is an associative algebra in $\mathcal{L} \mathcal{M}$.

- Let $B$ be the square-zero extension of an associative algebra $A$ by the $A$-module $M$. Then $(M \hookrightarrow B)$ is an algebra object in $\mathcal{L} \mathcal{M}$.

- Let $A$ be a Poisson algebra with bracket $\{-,-\}$, and let $\Omega^{1}(A)$ be the $A$-module of Kähler differentials over $A$. The pair $\left(A, \Omega^{1}(A)\right)$ is a Lie-Rinehart algebra with anchor map $\rho: \Omega^{1}(A) \rightarrow \operatorname{Der}(A)$ given by $d a \mapsto\{a,-\}$ for all $a \in A$ and Lie algebra structure on $\Omega^{1}(A)$ given by $[d a, d b]_{\Omega^{1}(A)}:=d\{a, b\}$. The differential map $d: A \rightarrow \Omega^{1}(A)$ is a Lie algebra object in $\mathcal{L} \mathcal{M}$, where $A$ is a right $\Omega^{1}(A)$-module with action $A \otimes \Omega^{1}(A) \rightarrow A$ given by $a \otimes b \cdot d c \mapsto b \cdot\{a, c\}$ for all $a, b, c \in A$. 
- Similarly, let $A$ be a Jacobi algebra with bracket $\{-,-\}_{J}$, and let $\mathcal{J}^{1}(A)$ be its 1 -jet space. Then the pair $\left(A, \mathcal{J}^{1}(A)\right)$ is a Lie-Rinehart algebra (see [22] for more details), and the map $j: A \rightarrow \mathcal{J}^{1}(A)$ given by $a \mapsto j^{1}(a)$ is a Lie algebra object in $\mathcal{L} \mathcal{M}$ with right $\mathcal{J}^{1}(A)$-action on $A$ given by $a \otimes b \cdot j^{1}(c) \mapsto b \cdot\{a, c\}_{J}$ for all $a, b, c \in A$.

Note that a Lie algebra object $(N \stackrel{f}{\rightarrow} L)$ in $\mathcal{L} \mathcal{M}$ is a very similar object to a strict 2 -term $L_{\infty}$ algebra.

We now focus on the description of $(M \stackrel{g}{\rightarrow} A)$-modules in $\mathcal{L} \mathcal{M}$ :

Proposition 3.6. A left $(M \stackrel{g}{\rightarrow} A)$-module object is a map $(V \stackrel{u}{\rightarrow} W)$ of left $A$-modules such that there exists an A-module map $\mu_{1}: W \otimes M \rightarrow V$ satisfying $g \circ \mu_{1}(m \otimes w)=\mu_{0}(g(m) \otimes w)$ for $w \in W$ and $m \in M$, which descends to an A-module map $\alpha_{\ell}^{V}: M \otimes_{A} W \rightarrow V$ (called structure map of the left $(M \stackrel{g}{\rightarrow} A)$-module $(V \stackrel{u}{\rightarrow} W))$ satisfying

$$
u \circ \alpha_{\ell}^{V}\left(m \otimes_{A} w\right)=\mu_{0} \circ\left(g(m) \otimes_{A} w\right) .
$$

Proof. Since $(V \stackrel{u}{\rightarrow} W)$ is a left $(M \stackrel{g}{\rightarrow} A)$-module, there exists a morphism

$$
\mu:(M \stackrel{g}{\rightarrow} A) \otimes(V \stackrel{u}{\rightarrow} W) \longrightarrow(V \stackrel{u}{\rightarrow} W),
$$

that is, a commuting square

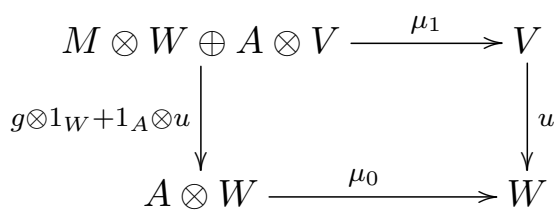

where $\mu_{1}$ and $\mu_{0}$ satisfy some associativity conditions. From (3.4) we see that $\mu_{0}$ and the restriction of $\mu_{1}$ to $A \otimes V$ turn $W$ and $V$ respectively into left $A$-modules, so that the vertical map $u$ becomes a map of left $A$-modules. Now, since $M$ is an $A$-bimodule and $W$ is a left $A$-module, we can construct the tensor product $M \otimes_{A} W$. Moreover, by the associativity of the module action $\mu$, we deduce that $\mu_{1}$ vanishes on $m \cdot a \otimes w-m \otimes a \cdot w$ where $m \in M, a \in A$, $w \in W$ so that the map $\mu_{1}: M \otimes W \rightarrow V$ descends to a map $\alpha_{\ell}^{V}: M \otimes_{A} W \rightarrow V$ yielding the following diagram:

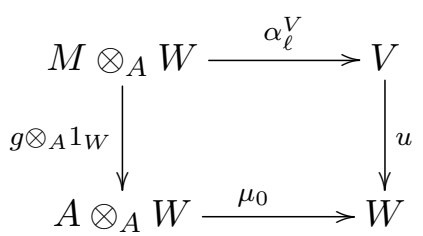

The commutativity of (3.5) ensures that the compatibility relation (3.3) is satisfied.

Proposition 3.7. For any Lie algebra object $(N \stackrel{f}{\rightarrow} L)$ endowed with an $(M \stackrel{g}{\rightarrow} A)$-module structure, the Leibniz bracket on $N$ given by $\left[n_{1}, n_{2}\right]_{N}:=\left[n_{1}, f\left(n_{2}\right)\right]$ satisfies

$$
\left[n_{1}, a \cdot\left[n_{2}, n_{2}\right]_{N}\right]_{N}=0, \quad \forall a \in A .
$$

Proof. By definition, a Lie algebra object $(N \stackrel{f}{\rightarrow} L)$ carries a Leibniz algebra structure on the $L$-module $N$ with bracket given by $\left[n_{1}, n_{2}\right]_{N}:=\left[n_{1}, f\left(n_{2}\right)\right]$ so that $\left[n_{1}, n_{2}\right]_{N}=0$ for all $n_{2} \in \operatorname{Ker}(f)$. Now, since $(N \stackrel{f}{\rightarrow} L)$ is an $(M \stackrel{g}{\rightarrow} A)$-module, $N$ and $L$ are $A$-modules and the vertical map $f$ is $A$-linear, we deduce that $\operatorname{Ker}(f)$ is an $A$-module. Hence $[-,-]_{N}$ satisfies condition (3.6). 


\subsection{The Lie algebra of derivations in $\mathcal{L} \mathcal{M}$}

In this section we describe the Lie algebra of derivations of an associative algebra object in $\mathcal{L} \mathcal{M}$. We start by describing morphisms between Lie algebra objects and Lie algebra actions.

Proposition 3.8. An algebra morphism $\phi:(M \stackrel{g}{\rightarrow} A) \rightarrow\left(M^{\prime} \stackrel{g^{\prime}}{\rightarrow} A^{\prime}\right)$ in $\mathcal{L} \mathcal{M}$ is given by a pair of maps $\left(\phi_{1}, \phi_{0}\right)$ satisfying

$$
\begin{aligned}
& \phi_{1}\left(a_{1} m_{1}+m_{2} a_{2}\right)=\phi_{0}\left(a_{1}\right) \phi_{1}\left(m_{1}\right)+\phi_{1}\left(m_{2}\right) \phi_{0}\left(a_{1}\right), \\
& \phi_{0}\left(a_{1} \cdot a_{2}\right)=\phi_{0}\left(a_{1}\right) \cdot \phi_{0}\left(a_{2}\right)
\end{aligned}
$$

for $a_{1}, a_{2} \in A$ and $m_{1}, m_{2} \in M$.

Proof. Assume $\phi:=\left(\phi_{1}, \phi_{0}\right)$ is an algebra morphism (in $\left.\mathcal{L} \mathcal{M}\right)$. Then using (3.1) and (3.2), a straightforward computation shows

$$
0=\phi \circ \mu-\mu \circ(\phi \otimes \phi)=\left(\phi_{1} \circ \mu_{1}-\mu_{1} \circ\left(\phi_{1} \otimes \phi_{0}\right), \phi_{0} \circ \mu_{0}-\mu_{0} \circ\left(\phi_{0} \otimes \phi_{0}\right)\right),
$$

that is

$$
\phi_{1} \circ \mu_{1}-\mu_{1} \circ\left(\phi_{1} \otimes \phi_{0}\right)=0, \quad \phi_{0} \circ \mu_{0}-\mu_{0} \circ\left(\phi_{0} \otimes \phi_{0}\right)=0
$$

which yield the relations in (3.7) and (3.8).

Proposition 3.9. A Lie algebra map a: $(N \stackrel{f}{\rightarrow} L) \rightarrow\left(N^{\prime} \stackrel{f^{\prime}}{\rightarrow} L^{\prime}\right)$ in $\mathcal{L} \mathcal{M}$ is given by a pair of maps $\left(a_{1}, a_{0}\right)$ satisfying

$$
a_{1}([n, \xi])=\left[a_{1}(n), a_{0}(\xi)\right], \quad a_{0}\left([\xi, \zeta]_{L}\right)=\left[a_{0}(\xi), a_{0}(\zeta)\right]_{L^{\prime}}
$$

for $n \in N$ and $\xi, \zeta \in L$.

Proof. An identical argument as in the proof for Proposition 3.8 and abusing notation so that $\mu$ is the Lie bracket on $(N \stackrel{f}{\rightarrow} L)$ we obtain the relation in (3.9) which, in this case, yields the relations in (3.10).

Proposition 3.10. Given a Lie algebra object $(N \stackrel{f}{\rightarrow} L)$, a left $(N \stackrel{f}{\rightarrow} L)$-module $($ in $\mathcal{L} \mathcal{M})$ is an object $(V \stackrel{u}{\rightarrow} W)$ such that $V$ and $W$ are left $L$-modules with actions given by

$$
\alpha_{0}: L \otimes W \longrightarrow W, \quad \alpha_{2}: L \otimes V \longrightarrow V
$$

and there exists an R-linear map

$$
\alpha_{1}: N \otimes W \longrightarrow V
$$

satisfying the following compatibility condition

$$
\alpha_{1}([n, \xi] \otimes w)=\alpha_{1}\left(n \otimes \alpha_{0}(\xi \otimes w)\right)-\alpha_{2}\left(\xi \otimes \alpha_{1}(n \otimes w)\right) .
$$

Moreover, the following compatibility conditions between $u, f, \alpha_{1}, \alpha_{2}$ and $\alpha_{3}$ are satisfied

$$
u \circ \alpha_{1}=\alpha_{0} \circ\left(f \otimes 1_{W}\right), \quad u \circ \alpha_{2}=\alpha \circ\left(1_{L} \otimes u\right) .
$$


Proof. Since $(V \stackrel{u}{\rightarrow} W)$ is a left $(N \stackrel{f}{\rightarrow} L)$-module, we have a morphism

$$
\alpha:(N \stackrel{f}{\rightarrow} L) \otimes(V \stackrel{u}{\rightarrow} W) \longrightarrow(V \stackrel{u}{\rightarrow} W)
$$

that is, a pair of maps $\left(\alpha_{1}+\alpha_{2}, \alpha_{0}\right)$ where $\alpha_{0}, \alpha_{1}, \alpha_{2}$ are given by the maps in (3.11) and (3.12), such that the diagram commutes

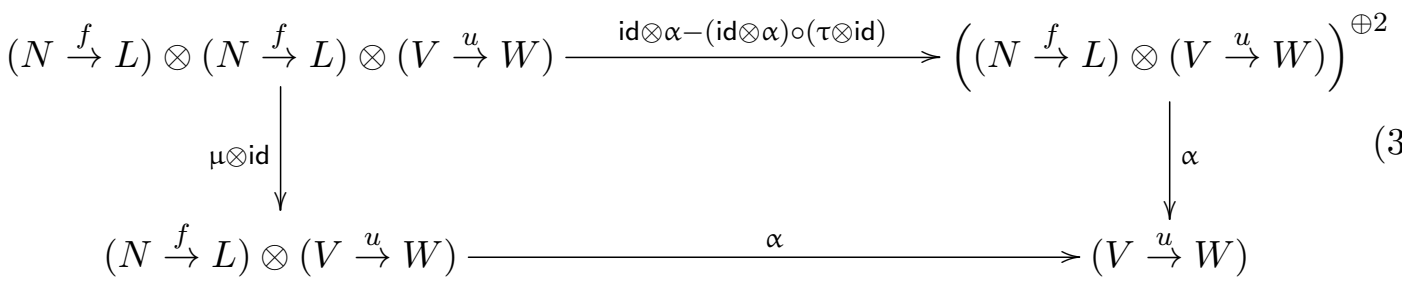

which can be seen as the following diagram in cube shape

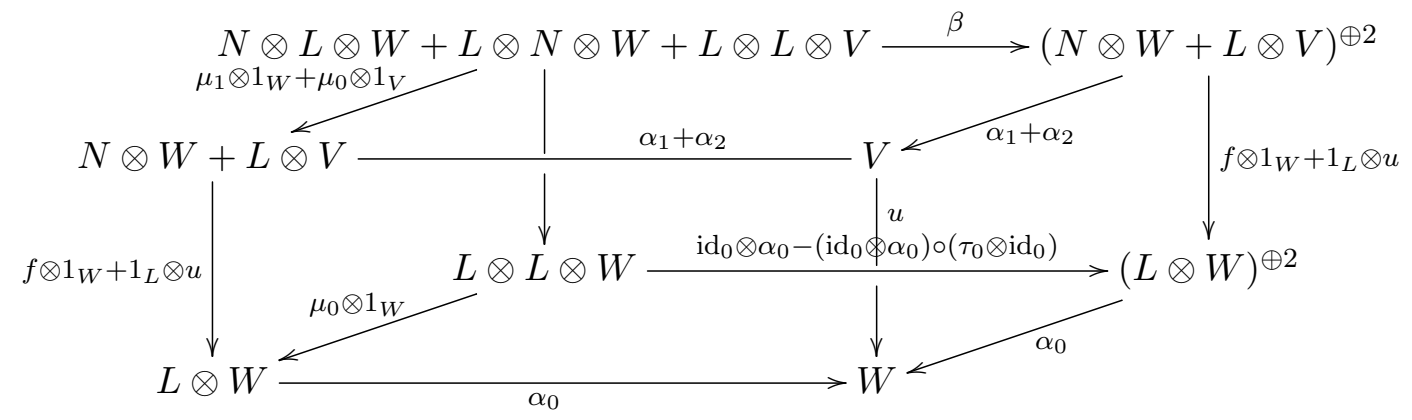

where

$$
\begin{aligned}
\beta:= & \mathrm{id}_{1} \otimes \alpha_{0}-\left(\mathrm{id}_{0} \otimes \alpha_{1}\right) \circ\left(\tau_{1} \otimes \mathrm{id}_{0}\right)+\mathrm{id}_{0} \otimes \alpha_{1} \\
& -\left(\mathrm{id}_{1} \otimes \alpha_{0}\right) \circ\left(\tau_{1} \otimes \mathrm{id}_{0}\right)+\mathrm{id}_{0} \otimes \alpha_{2}-\left(\mathrm{id}_{0} \otimes \alpha_{2}\right) \circ\left(\tau_{0} \otimes \mathrm{id}_{1}\right) .
\end{aligned}
$$

Using (3.1) and (3.2), a long but straightforward computation shows that the compatibility relation making (3.14) commute can be expressed as

$$
\begin{aligned}
0= & \alpha \circ(\mathrm{id} \otimes \alpha-(\mathrm{id} \otimes \alpha) \circ(\tau \otimes \mathrm{id}))-\alpha \circ(\mu \otimes \mathrm{id}) \\
= & \left(\alpha_{1} \circ\left(\mathrm{id}_{1} \otimes \alpha_{0}\right)-\alpha_{2} \circ\left(\mathrm{id}_{0} \otimes \alpha_{1}\right) \circ\left(\tau_{1} \otimes \mathrm{id}_{0}\right)+\alpha_{2} \circ\left(\mathrm{id}_{0} \otimes \alpha_{1}\right)\right. \\
& -\alpha_{1} \circ\left(\mathrm{id}_{1} \otimes \alpha_{0}\right) \circ\left(\tau_{1} \otimes \mathrm{id}_{0}\right)+\alpha_{2} \circ\left(\mathrm{id}_{0} \otimes \alpha_{2}\right)-\alpha_{2} \circ\left(\mathrm{id}_{0} \otimes \alpha_{2}\right) \circ\left(\tau_{0} \otimes \mathrm{id}_{1}\right), \\
& \left.\alpha_{0} \circ\left(\mathrm{id}_{0} \otimes \alpha_{0}\right)-\alpha_{0} \circ\left(\mathrm{id}_{0} \otimes \alpha_{0}\right) \circ\left(\tau_{0} \otimes \mathrm{id}_{0}\right)\right) \\
& -\left(\alpha_{1} \circ\left(\mu_{1} \otimes \mathrm{id}_{0}\right)+\alpha_{2} \circ\left(\mu_{0} \otimes \mathrm{id}_{1}\right), \alpha_{0} \circ\left(\mu_{0} \otimes \mathrm{id}_{0}\right)\right) \\
= & \left(\alpha_{1} \circ\left(\mathrm{id}_{1} \otimes \alpha_{0}\right)-\alpha_{2} \circ\left(\mathrm{id}_{0} \otimes \alpha_{1}\right) \circ\left(\tau_{1} \otimes \mathrm{id}_{0}\right)-\alpha_{1} \circ\left(\mu_{1} \otimes \mathrm{id}_{0}\right)\right. \\
& +\left(\alpha_{2} \circ\left(\mathrm{id}_{0} \otimes \alpha_{1}\right)-\alpha_{1} \circ\left(\mathrm{id}_{1} \otimes \alpha_{0}\right) \circ\left(\tau_{1} \otimes \mathrm{id}_{0}\right)-\alpha_{1} \circ\left(\mu_{1} \otimes \mathrm{id}_{0}\right)\right. \\
& +\left(\alpha_{2} \circ\left(\mathrm{id}_{0} \otimes \alpha_{2}\right)-\alpha_{2} \circ\left(\mathrm{id}_{0} \otimes \alpha_{2}\right) \circ\left(\tau_{0} \otimes \mathrm{id}_{1}\right)-\alpha_{2} \circ\left(\mu_{0} \otimes \mathrm{id}_{1}\right),\right. \\
& \left.\alpha_{0} \circ\left(\mathrm{id}_{0} \otimes \alpha_{0}\right)-\alpha_{0} \circ\left(\mathrm{id}_{0} \otimes \alpha_{0}\right) \circ\left(\tau_{0} \otimes \mathrm{id}_{0}\right)-\alpha_{0} \circ\left(\mu_{0} \otimes \mathrm{id}_{0}\right)\right),
\end{aligned}
$$

so that the following diagrams commute

- A diagram encoding the $L$-module action $\alpha_{0}: L \otimes W \rightarrow W$

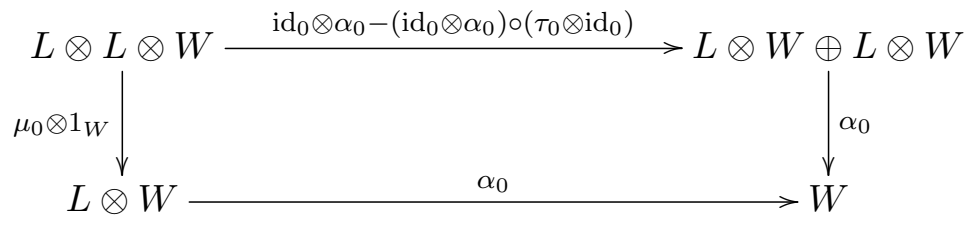


- A diagram encoding the $L$-module action $\alpha_{2}: L \otimes V \rightarrow V$

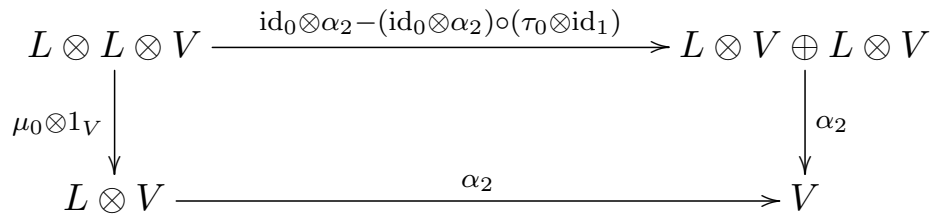

- And lastly

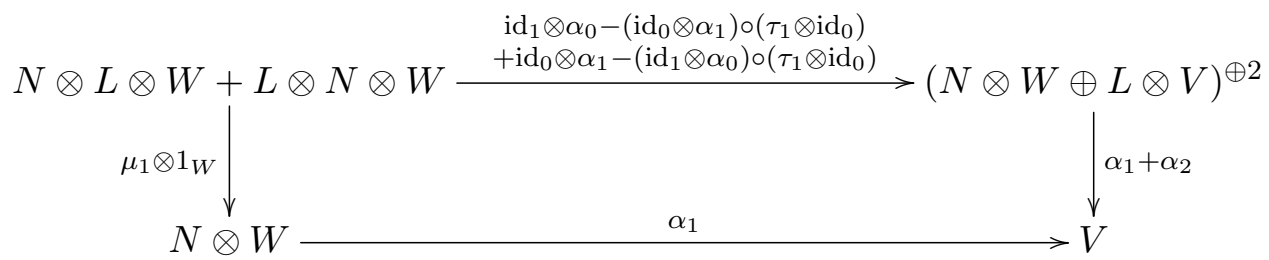

encoding the compatibility relation in (3.12).

By the adjoint functor property of tensor products, the maps in (3.11) correspond to

$$
\alpha_{0}: L \rightarrow \operatorname{Hom}_{R}(W, W), \quad \alpha_{1}: \quad N \rightarrow \operatorname{Hom}_{R}(W, V), \quad \alpha_{2}: L \rightarrow \operatorname{Hom}_{R}(V, V)
$$

that we can describe as the following commutative diagram

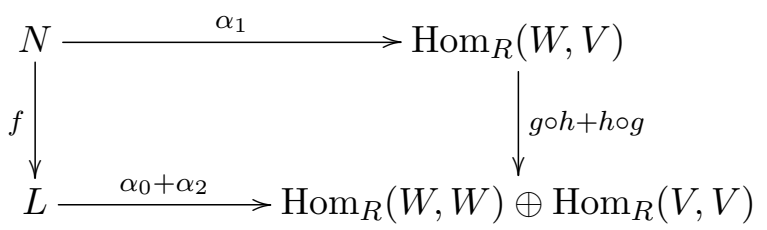

where $h \in \operatorname{Hom}_{R}(W, V)$.

Proposition 3.11. Let $(M \stackrel{g}{\rightarrow} A)$ be a commutative algebra object in $\mathcal{L} \mathcal{M}$. A Lie algebra object $(N \stackrel{f}{\rightarrow} L)$ is said to act on $(M \stackrel{g}{\rightarrow} A)$ by derivations if there exist two Lie algebra maps

$$
\rho_{0}: L \rightarrow \operatorname{Der}_{R}(A), \quad \rho_{2}: L \rightarrow H:=\left(\operatorname{Hom}_{R}(M, M),[-,-]_{H}\right)
$$

satisfying the compatibility conditions

$$
\rho_{2}(\xi)(a \cdot m)=a \cdot \rho_{2}(\xi)(m)+\rho_{0}(\xi)(a) \cdot m, \quad g\left(\rho_{2}(\xi)(m)\right)=\rho_{0}(\xi)(g(m))
$$

and an $R$-module map

$$
\rho_{1}: \quad N \rightarrow \operatorname{Der}_{R}(A, M)
$$

satisfying

$$
\rho_{1}([n, \xi])=\left[\rho_{1}(n),\left(\rho_{0}+\rho_{2}\right)(\xi)\right], \quad g\left(\rho_{1}(n)(a)\right)=\rho_{0}(f(n))(a)
$$

for all $\xi \in L, a \in A$ and $m \in M$. 
Proof. Let a Lie algebra $(N \stackrel{f}{\rightarrow} L)$ act on $(M \stackrel{g}{\rightarrow} A)$ by derivations, then there exists a left $(N \stackrel{f}{\rightarrow} L)$-module structure on $(M \stackrel{g}{\rightarrow} A)$, denoted by $\rho:(N \stackrel{f}{\rightarrow} L) \otimes(M \stackrel{g}{\rightarrow} A) \rightarrow(M \stackrel{g}{\rightarrow} A)$, which by Proposition 3.10 endows $M$ and $A$ with left $L$-actions given by $\varrho_{0}: L \otimes A \rightarrow A$ and $\varrho_{2}: L \otimes M \rightarrow M$ respectively, and induces a map $\varrho_{1}: N \otimes A \rightarrow M$ satisfying (3.13). Furthermore, the action of $(N \stackrel{f}{\rightarrow} L)$ on $(M \stackrel{g}{\rightarrow} A)$ by derivations makes the following commute

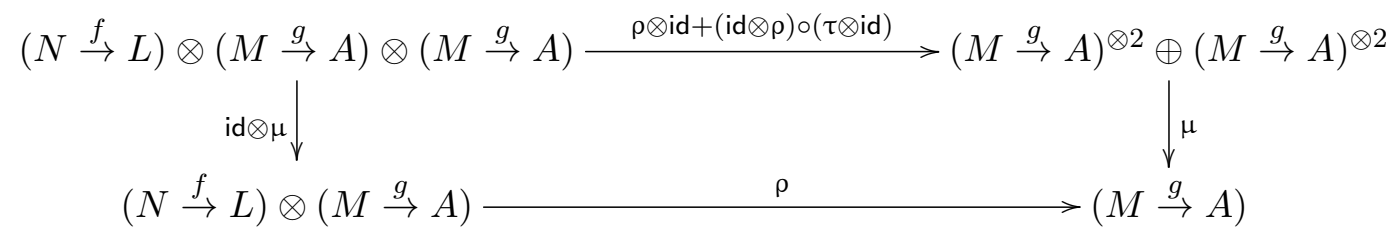

Since $\rho=\left(\varrho_{1}+\varrho_{2}, \varrho_{0}\right)$, by $(3.1)$ and $(3.2)$ we find

$$
\begin{aligned}
0= & \mu \circ(\rho \otimes \mathrm{id}+(\mathrm{id} \otimes \rho) \circ(\tau \otimes \mathrm{id}))-\rho \circ(\mathrm{id} \otimes \mu) \\
= & \left(\mu_{1} \circ\left(\varrho_{1} \otimes \mathrm{id}_{0}\right)+\mu_{1} \circ\left(\mathrm{id}_{0} \otimes \varrho_{1}\right) \circ\left(\tau_{0} \otimes \mathrm{id}_{1}\right)-\rho_{1} \circ\left(\mathrm{id}_{1} \otimes \mu_{0}\right)+\mu_{1} \circ\left(\varrho_{2} \otimes \mathrm{id}_{0}\right)\right. \\
& +\mu_{1} \circ\left(\mathrm{id}_{1} \otimes \varrho_{0}\right) \circ\left(\tau_{1} \otimes \mathrm{id}_{0}\right)+\mu_{1} \circ\left(\varrho_{0} \circ \mathrm{id}_{1}\right)+\mu_{1} \circ\left(\mathrm{id}_{0} \otimes \varrho_{2}\right) \circ\left(\tau_{1} \otimes \mathrm{id}_{0}\right) \\
& \left.-\varrho_{2} \circ\left(\mathrm{id}_{1} \otimes \mu_{0}\right), \mu_{0} \circ\left(\varrho_{0} \otimes \mathrm{id}_{0}\right)+\mu_{0} \circ\left(\mathrm{id}_{0} \otimes \varrho_{0}\right) \circ\left(\tau_{0} \otimes \mathrm{id}_{0}\right)-\varrho_{0} \circ\left(\mathrm{id}_{0} \otimes \mu_{0}\right)\right),
\end{aligned}
$$

so the following diagrams commute:

- a diagram which encodes the universal action of a Lie algebra $L$ on $A$ by derivations

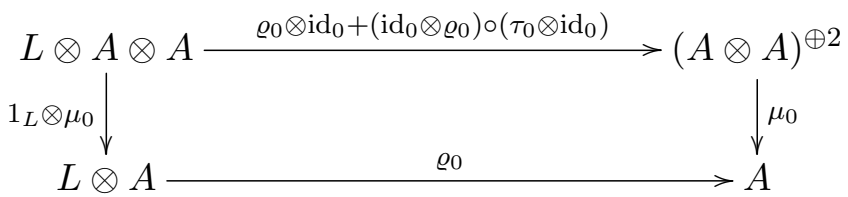

- a diagram encoding the action of $N$ on $A$

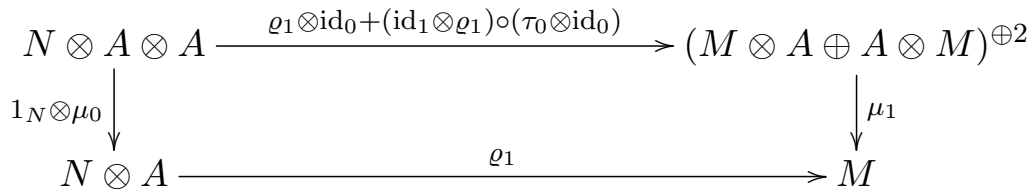

- and lastly, a commutative diagram encoding the action of $L$ on both $A$ and $M$

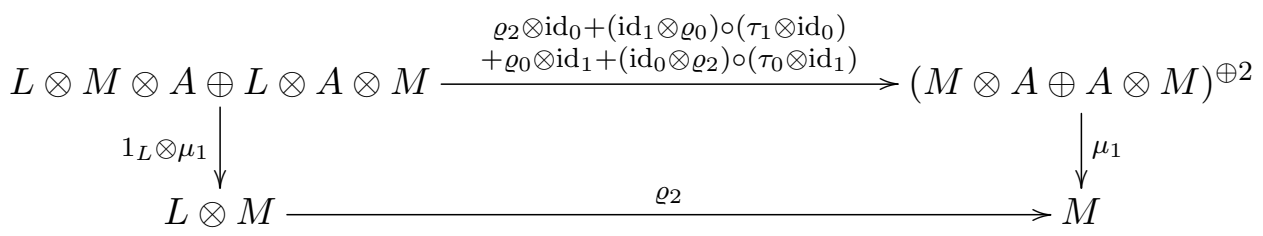

These maps make the following cube commute

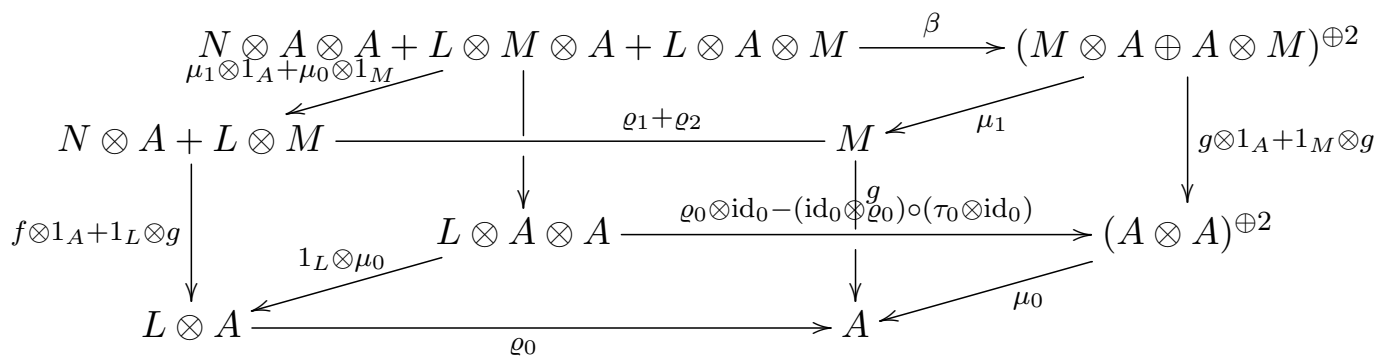


By the adjoint functor property of tensor products, the maps $\varrho_{1}, \varrho_{2}$ and $\varrho_{3}$ are equivalent to the maps in (3.16).

Proposition 3.12. Recall $(M \stackrel{f}{\rightarrow} A)$ is a commutative algebra object and $H=\operatorname{Hom}_{R}(M, M)$. Let the $R$-module maps $\pi_{1}: \operatorname{Der}_{R}(A, M) \rightarrow \operatorname{Der}_{R}(A), \pi_{2}: \operatorname{Der}_{R}(A, M) \rightarrow H$ be given by $\pi_{1}(\partial):=$ $g \circ \partial$ and $\pi_{2}(\partial):=\partial \circ g$ respectively. The universal Lie algebra of derivations of the algebra object $(M \stackrel{g}{\rightarrow} A)$ is given by

$$
\operatorname{Der}_{\mathcal{L M}}((M \stackrel{g}{\rightarrow} A))=\left(\operatorname{Der}_{R}(A, M) \stackrel{\pi_{1}+\pi_{2}}{\longrightarrow} \operatorname{Der}_{R}(A) \oplus H\right)
$$

where the Lie bracket on $\operatorname{Der}_{R}(A) \oplus H$ is

$$
\left[(\alpha, \beta),\left(\alpha^{\prime}, \beta^{\prime}\right)\right]_{\operatorname{Der}_{R}(A) \oplus H}:=\left(\left[\alpha, \alpha^{\prime}\right]_{\operatorname{Der}_{R}(A)},-\left[\beta, \beta^{\prime}\right]_{H}\right),
$$

and the right $\operatorname{Der}_{R}(A) \oplus H$-module structure on $\operatorname{Der}_{R}(A, M)$ is given by

$$
\partial \otimes(\alpha, \beta) \longmapsto[\partial,(\alpha, \beta)]:=\partial \circ \alpha-\beta \circ \partial .
$$

Proof. We first check that the action $[-,-]$ in (3.19) endows $\operatorname{Der}_{R}(A, M)$ with a right Lie algebra module structure over (the Lie algebra) $\operatorname{Der}_{R}(A) \oplus H$.

On the one hand we have

$$
\begin{aligned}
{\left[\partial,\left[(\alpha, \beta),\left(\alpha^{\prime}, \beta^{\prime}\right)\right]_{\operatorname{Der}_{R}(A) \oplus H}\right] } & =\left[\partial,\left(\left[\alpha, \alpha^{\prime}\right]_{\operatorname{Der}_{R}(A)},\left[\beta, \beta^{\prime}\right]_{H}\right)\right] \\
& =\partial \circ\left[\alpha, \alpha^{\prime}\right]_{\operatorname{Der}_{R}(A)}-\left[\beta, \beta^{\prime}\right]_{H} \circ \partial .
\end{aligned}
$$

On the other hand we have

$$
\begin{aligned}
{\left[[\partial,(\alpha, \beta)],\left(\alpha^{\prime}, \beta^{\prime}\right)\right] } & =\left[(\partial \circ \alpha-\beta \circ \partial),\left(\alpha^{\prime}, \beta^{\prime}\right)\right] \\
& =(\partial \circ \alpha-\beta \circ \partial) \circ \alpha^{\prime}-\beta^{\prime} \circ(\partial \circ \alpha-\beta \circ \partial) \\
& =\partial \circ \alpha \circ \alpha^{\prime}-\beta \circ \partial \circ \alpha^{\prime}-\beta^{\prime} \circ \partial \circ \alpha+\beta^{\prime} \circ \beta \circ \partial,
\end{aligned}
$$

so that

$$
\begin{aligned}
& {\left[[\partial,(\alpha, \beta)],\left(\alpha^{\prime}, \beta^{\prime}\right)\right]-\left[\left[\partial,\left(\alpha^{\prime}, \beta^{\prime}\right)\right],(\alpha, \beta)\right]} \\
& \quad=\partial \circ \alpha \circ \alpha^{\prime}-\beta \circ \partial \circ \alpha^{\prime}-\beta^{\prime} \circ \partial \circ \alpha+\beta^{\prime} \circ \beta \circ \partial-\partial \circ \alpha^{\prime} \circ \alpha \\
& \quad+\beta^{\prime} \circ \partial \circ \alpha+\beta \circ \partial \circ \alpha^{\prime}-\beta \circ \beta^{\prime} \circ \partial \\
& \quad=\partial \circ\left[\alpha, \alpha^{\prime}\right]_{\operatorname{Der}_{R}(A)}-\left[\beta, \beta^{\prime}\right]_{H} \circ \partial=\left[\partial,\left(\left[\alpha, \alpha^{\prime}\right]_{\operatorname{Der}_{R}(A)},\left[\beta, \beta^{\prime}\right]_{H}\right)\right] .
\end{aligned}
$$

This shows that the right action of $\operatorname{Der}_{R}(A) \oplus H$ on $\operatorname{Der}_{R}(A, M)$ is well defined. Hence, the morphism $\rho:(N \stackrel{f}{\rightarrow} L) \rightarrow \operatorname{Der}_{\mathcal{L M}}((M \stackrel{g}{\rightarrow} A))$ is given by the following diagram, which follows from $(3.15)$

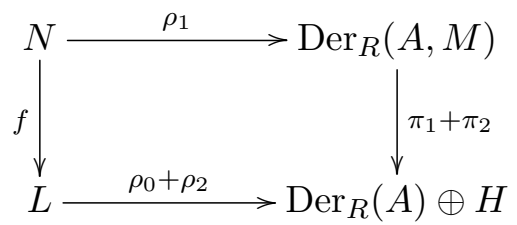

which commutes.

Remark 3.13. The morphism $\rho:(N \stackrel{f}{\rightarrow} L) \rightarrow \operatorname{Der}_{\mathcal{L M}}((M \stackrel{g}{\rightarrow} A))$ given by $\left(\rho_{1}, \rho_{0}+\rho_{2}\right)$ is a morphism of Lie algebras in $\mathcal{L M}$. 
Example 3.14. The universal Lie algebra of derivations of the commutative algebra object $(A \stackrel{\text { id }}{\rightarrow} A)$ is

$$
\left(\operatorname{Der}_{R}(A) \longrightarrow \operatorname{Der}_{R}(A) \oplus \operatorname{Der}_{R}(A)\right) \text {. }
$$

Then, the action of a Lie algebra object $(N \stackrel{f}{\rightarrow} L)$ by derivations on $(A \stackrel{\text { id }}{\rightarrow} A)$ is given by

- a Lie algebra map $\rho_{0} \equiv \rho_{2}: L \rightarrow \operatorname{Der}_{R}(A)$,

- an $A$-module map $\rho_{1}: N \rightarrow \operatorname{Der}_{R}(A)$

satisfying $\rho_{1}(n)=\rho_{0}(f(n))$.

\subsection{Lie-Rinehart algebra objects in $\mathcal{L} \mathcal{M}$}

A Lie-Rinehart algebra [8, 21] is an algebraic structure which encompasses a Lie algebra and a commutative algebra which act on each other in a way that both actions are compatible. This object can be described in any symmetric monoidal category.

In this Section we focus on the description of Lie-Rinehart algebra objects in the category $\mathcal{L} \mathcal{M}$ of linear maps. Based on [19, Lemma 3.6] we give a proof of Theorem 1.1.

Proof of Theorem 1.1. Assume the pair $((M \stackrel{g}{\rightarrow} A),(N \stackrel{f}{\rightarrow} L))$ is a Lie-Rinehart algebra object. Then there exist

- a left $(M \stackrel{g}{\rightarrow} A)$-module structure on the Lie algebra object $(N \stackrel{f}{\rightarrow} L)$,

- an action $\rho$ of the Lie algebra object $(N \stackrel{f}{\rightarrow} L)$ on the commutative algebra object $(M \stackrel{g}{\rightarrow} A)$ by derivations,

and a compatibility condition between these two actions. We now describe what these structures involve.

Firstly, by Proposition 3.6, we deduce that a left $(M \stackrel{g}{\rightarrow} A)$-module structure on the Lie algebra object $(N \stackrel{f}{\rightarrow} L)$ turns the $L$-equivariant map $f: N \rightarrow L$ into an $A$-module map. Also, it yields an $A$-module map $\lambda:=\alpha_{\ell}^{N}: M \otimes_{A} L \rightarrow N$. Moreover the Leibniz algebra structure on $N$ given by $[-,-]_{N}$ must satisfy $\left[n_{1}, a \cdot\left[n_{2}, n_{2}\right]_{N}\right]_{N}=0$ for all $a \in A$ and $n_{1}, n_{2} \in N$.

Secondly, by Proposition 3.11, an action $\rho$ of the Lie algebra object $(N \stackrel{f}{\rightarrow} L)$ on the commutative algebra $(M \stackrel{g}{\rightarrow} A)$ by derivations yields

- an $A$-linear Lie algebra map $\rho_{0}: L \rightarrow \operatorname{Der}_{R}(A)$,

- an $A$-module map $\rho_{1}: N \rightarrow \operatorname{Der}_{R}(A, M)$,

- an $A$-linear Lie algebra map $\rho_{2}: L \rightarrow \operatorname{Hom}_{R}(M, M)$

satisfying conditions (3.17), (3.18), and turns $g: M \rightarrow A$ into an $L$-equivariant map. Lastly, the following compatibility conditions between the two module structures are satisfied:

- the pair $(A, L)$ is a Lie-Rinehart algebra with anchor $\rho_{0}$,

- the right $L$-action on the $A$-module $N$ satisfies

$$
[a \cdot n, b \cdot \xi]=a \cdot[n, b \cdot \xi]-b \cdot \rho_{0}(\xi)(a) \cdot n,
$$

which provides a compatibility relation between the right $L$-action on $N$, given by $[-,-]$ and the $A$-module structure on $N$.

Note that $\rho_{2}$ endows $M$ with is a left $(A, L)$-module structure, see [9] for further details. 
Example 3.15. Let $(A, L)$ be a Lie-Rinehart algebra with anchor $\rho_{L}: L \rightarrow \operatorname{Der}_{R}(A)$. The pair $((A \stackrel{\text { id }}{\rightarrow} A),(L \stackrel{\text { id }}{\rightarrow} L))$ is a Lie-Rinehart algebra object in $\mathcal{L} \mathcal{M}$ with $\rho_{0} \equiv \rho_{1} \equiv \rho_{2}=\rho_{L}$ and $\lambda \equiv \mathrm{id}$.

Example 3.16. The pair $\left((M \stackrel{g}{\rightarrow} A),\left(\operatorname{Der}_{R}(A, M) \stackrel{\pi_{1}+\pi_{2}}{\longrightarrow} \operatorname{Der}_{R}(A) \oplus H\right)\right)$ is a Lie-Rinehart algebra object in $\mathcal{L} \mathcal{M}$ with

$$
\begin{aligned}
& \rho_{0}: \operatorname{Der}_{R}(A) \oplus H \rightarrow \operatorname{Der}_{R}(A), \quad \rho_{1}: \operatorname{Der}_{R}(A, M) \rightarrow \operatorname{Der}_{R}(A, M), \\
& \rho_{2}: \operatorname{Der}_{R}(A) \oplus H \rightarrow H .
\end{aligned}
$$

\section{Leibniz algebroids and Lie-Rinehart algebras in $\mathcal{L M}$}

In this short section we prove Theorem 1.2. This result provides a functorial relation from Lie-Rinehart algebras to Leibniz algebroids.

Proof of Theorem 1.2. First note that $N$ is both a right $L$-module and a left $A$-module. Also, recall from [19] that the right $L$-module $N$ becomes a Leibniz algebra by defining the bracket $\left[n_{1}, n_{2}\right]_{N}=\left[n_{1}, f\left(n_{2}\right)\right]$. Furthermore, since $M$ is a left $(A, L)$-module, by Proposition 2.6 we can endow the $A$-module $M \oplus N$ with a Leibniz algebra structure given by the bracket (1.2). Since $f: N \rightarrow L$ and $\rho_{0}: L \rightarrow \operatorname{Der}_{R}(A)$ are $A$-linear maps, the map $\rho_{M \oplus N}=-\rho_{0} \circ f$ is also $A$-linear. We now prove that $\rho_{M \oplus N}$ is a Leibniz algebra antihomomorphism

$$
\begin{aligned}
& \rho_{M \oplus N}\left(\left[m_{1}+n_{1}, m_{2}+n_{2}\right]_{M \oplus N}\right)=\rho_{M \oplus N}\left(-\rho_{2}\left(f\left(n_{2}\right)\right)\left(m_{1}\right)+\left[n_{1}, n_{2}\right]_{N}\right) \\
& \quad=-\rho_{0}\left(f\left(\left[n_{1}, f\left(n_{2}\right)\right]\right)\right)=-\rho_{0}\left(\left[f\left(n_{1}\right), f\left(n_{2}\right)\right]_{L}\right)=\left[\rho_{0}\left(f\left(n_{2}\right)\right), \rho_{0}\left(f\left(n_{1}\right)\right)\right]_{\operatorname{Der}_{R}(A)} \\
& \quad=\left[\rho_{M \oplus N}\left(n_{2}\right), \rho_{M \oplus N}\left(n_{1}\right)\right]_{\operatorname{Der}_{R}(A)},
\end{aligned}
$$

so the relations in (1.1) hold. We now prove that the Leibniz rule in (2.4) hold

$$
\begin{aligned}
& {\left[a \cdot\left(m_{1}+n_{1}\right), m_{2}+n_{2}\right]_{M \oplus N}=-\rho_{2}\left(f\left(n_{2}\right)\right)\left(a \cdot m_{1}\right)+\left[a \cdot n_{1}, f\left(n_{2}\right)\right]} \\
& \quad=-a \cdot \rho_{2}\left(f\left(n_{2}\right)\right) \cdot m_{1}-\rho_{0}\left(f\left(n_{2}\right)\right)(a) \cdot m_{1}+a \cdot\left[n_{1}, f\left(n_{2}\right)\right]-\rho_{0}\left(f\left(n_{2}\right)\right)(a) \cdot n_{1} \\
& \quad=a \cdot\left(-\rho_{2}\left(f\left(n_{2}\right)\right) \cdot m_{1}+\left[n_{1}, f\left(n_{2}\right)\right]\right)+\rho_{M \oplus N}(a) \cdot\left(m_{1}+n_{1}\right) \\
& \quad=a \cdot\left[m_{1}+n_{1}, m_{2}+n_{2}\right]_{M \oplus N}+\rho_{M \oplus N}\left(m_{2}+n_{2}\right)(a) \cdot\left(m_{1}+n_{1}\right) .
\end{aligned}
$$

Example 4.1. Given the Lie-Rinehart algebra

$$
\left((M \stackrel{g}{\rightarrow} A),\left(\operatorname{Der}_{R}(A, M) \stackrel{\pi_{1}+\pi_{2}}{\longrightarrow} \operatorname{Der}_{R}(A) \oplus H\right)\right)
$$

in $\mathcal{L} \mathcal{M}$, the pairs $\left(A, \operatorname{Der}_{R}(A, M)\right)$ and $\left(A, M \oplus \operatorname{Der}_{R}(A, M)\right)$ are Leibniz algebroids.

Example 4.2. Given a Lie-Rinehart algebra $(A, L)$, the pair

$$
((A \stackrel{\text { id }}{\rightarrow} A),(L \stackrel{\text { id }}{\rightarrow} L))
$$

is a Lie-Rinehart algebra object in $\mathcal{L} \mathcal{M}$ with corresponding Leibniz algebroid given by $(A, A \oplus L)$ with structure presented in Proposition 2.9.

\section{Acknowledgements}

The author wishes to thank Uli Krähmer for helpful comments and suggestions. It is also a pleasure to thank Stuart White for his time, José Figueroa O'Farrill for conversations, and the anonymous referees for their comments and suggestions. This research was funded by an EPSRC DTA grant. 


\section{References}

[1] Baraglia D., Leibniz algebroids, twistings and exceptional generalized geometry, J. Geom. Phys. 62 (2012), 903-934, arXiv:1101.0856.

[2] Bloh A., On a generalization of the concept of Lie algebra, Dokl. Akad. Nauk SSSR 165 (1965), 471-473.

[3] Böhm G., Szlachányi K., Hopf algebroids with bijective antipodes: axioms, integrals, and duals, J. Algebra 274 (2004), 708-750, math.QA/0302325.

[4] Cuvier C., Homologie de Leibniz et homologie de Hochschild, C. R. Acad. Sci. Paris Sér. I Math. 313 (1991), 569-572.

[5] Figueroa-O'Farrill J.M., Deformations of 3-algebras, J. Math. Phys. 50 (2009), 113514, 27 pages, arXiv:0903.4871.

[6] Herz J.-C., Pseudo-algèbres de Lie. I, C. R. Acad. Sci. Paris 236 (1953), 1935-1937.

[7] Huebschmann J., Poisson cohomology and quantization, J. Reine Angew. Math. 408 (1990), 57-113.

[8] Huebschmann J., On the quantization of Poisson algebras, in Symplectic Geometry and Mathematical Physics (Aix-en-Provence, 1990), Progr. Math., Vol. 99, Birkhäuser Boston, Boston, MA, 1991, $204-233$.

[9] Huebschmann J., Lie--Rinehart algebras, Gerstenhaber algebras and Batalin-Vilkovisky algebras, Ann. Inst. Fourier (Grenoble) 48 (1998), 425-440, dg-ga/9704005.

[10] Ibáñez R., de León M., Marrero J.C., Padrón E., Leibniz algebroid associated with a Nambu-Poisson structure, J. Phys. A: Math. Gen. 32 (1999), 8129-8144, math-ph/9906027.

[11] Kinyon M.K., Weinstein A., Leibniz algebras, Courant algebroids, and multiplications on reductive homogeneous spaces, Amer. J. Math. 123 (2001), 525-550, math.DG/0006022.

[12] Kosmann-Schwarzbach Y., Courant algebroids. A short history, SIGMA 9 (2013), 014, 8 pages, arXiv:1212.0559.

[13] Krähmer U., Rovi A., A Lie-Rinehart algebra with no antipode, Comm. Algebra 43 (2015), $4049-4053$.

[14] Krähmer U., Wagemann F., Racks, Leibniz algebras and Yetter-Drinfel'd modules, Georgian Math. J., to appear, arXiv:1403.4148

[15] Kurdiani R., Pirashvili T., A Leibniz algebra structure on the second tensor power, J. Lie Theory 12 (2002), 583-596.

[16] Liu Z.-J., Weinstein A., Xu P., Manin triples for Lie bialgebroids, J. Differential Geom. 45 (1997), 547-574, dg-ga/9508013.

[17] Loday J.-L., Une version non commutative des algèbres de Lie: les algèbres de Leibniz, Enseign. Math. 39 (1993), 269-293.

[18] Loday J.-L., Pirashvili T., Universal enveloping algebras of Leibniz algebras and (co)homology, Math. Ann. 296 (1993), 139-158.

[19] Loday J.-L., Pirashvili T., The tensor category of linear maps and Leibniz algebras, Georgian Math. J. 5 (1998), 263-276.

[20] Pradines J., Théorie de Lie pour les groupoïdes différentiables. Calcul différenetiel dans la catégorie des groupoïdes infinitésimaux, C. R. Acad. Sci. Paris Sér. A-B 264 (1967), A245-A248.

[21] Rinehart G.S., Differential forms on general commutative algebras, Trans. Amer. Math. Soc. 108 (1963), 195-222.

[22] Rovi A., Hopf algebroids associated to Jacobi algebras, Int. J. Geom. Methods Mod. Phys. 11 (2014), 1450092, 20 pages, arXiv:1311.4181.

[23] Roytenberg D., Courant-Dorfman algebras and their cohomology, Lett. Math. Phys. 90 (2009), 311-351, arXiv:0902.4862.

[24] Stiénon M., Xu P., Modular classes of Loday algebroids, C. R. Math. Acad. Sci. Paris 346 (2008), 193-198, arXiv:0803.2047. 